\title{
A unified theory for irresolute functions
}

\author{
BISHWAMBHAR ROY
}

\begin{abstract}
In this paper, a new class called $(\mu, \lambda)_{\theta}$-irresolute functions has been defined with the notion of generalized topology. We obtain some characterizations of such functions and some relations between similar types of functions are established. Some basic properties of such functions are also discussed. Such functions unify different types of weakly irresolute functions by T. Noiri.
\end{abstract}

\section{Introduction}

In 1943, Fomin [8] introduced the concept of $\theta$-continuous functions. After that, different mathematicians continued the study of $\theta$-continuous functions (see $[7,11,12])$. The notion of $\theta$-irresolute functions was studied in $[5,6,9]$. The concept of $\theta$-preirresolute functions [13], weakly $\beta$-irresolute functions [14] and other similar types of functions have also been introduced and studied by different mathematicians. Our aim in this paper is to unify several properties and characterizations of the above mentioned functions or other similar types of functions by the notion of generalized topology [1] introduced by Császár.

After its introduction during the last ten years or so, the area of generalized topology is rapidly growing. Császár has published a series of papers introducing and studying generalized topology, generalized neighbourhood systems and generalized continuity, and has shown that the fundamental definitions and the major part of many statements and constructions in set topology can be formulated by replacing topology with the help of generalized topology.

We now recall some notions defined in [1]. Let $X$ be a non-empty set and $\exp X$ be the power set of $X$. We call a class $\mu \subseteq \exp X$ a generalized topology [1], (briefly, GT) if $\emptyset \in \mu$ and any union of elements of $\mu$ belongs to

Received May 28, 2021.

2020 Mathematics Subject Classification. 54C08, 54A05.

Key words and phrases. $\mu$-open sets, $\mu_{\theta}$-closure, $(\mu, \lambda)_{\theta}$-irresolute function.

https://doi.org/10.12697/ACUTM.2021.25.20 
$\mu$. A set $X$, with a GT $\mu$ on it is said to be a generalized topological space (briefly, GTS) and is denoted by $(X, \mu)$. For a GTS $(X, \mu)$, the elements of $\mu$ are called $\mu$-open sets, and the complements of $\mu$-open sets are called $\mu$-closed sets. For $A \subseteq X$, we denote by $c_{\mu}(A)$ the intersection of all $\mu$ closed sets containing $A$, i.e., the smallest $\mu$-closed set containing $A$; and by $i_{\mu}(A)$ the union of all $\mu$-open sets contained in $A$, i.e., the largest $\mu$-open set contained in $A$ (see $[2,4]$ ). A GTS $(X, \mu)$ is said to be a $\mu$-space [15] if $X \in \mu$.

It is easy to observe that $i_{\mu}$ and $c_{\mu}$ are idempotent and monotonic operators, where $\gamma: \exp X \rightarrow \exp X$ is said to be idempotent iff for each $A \subseteq X$, $\gamma(\gamma(A))=\gamma(A)$, and monotonic iff $\gamma(A) \subseteq \gamma(B)$ whenever $A \subseteq B \subseteq X$. It is also well known from $[4,2]$ that if $\mu$ is a GT on $X$ and $A \subseteq X, x \in X$, then $x \in c_{\mu}(A)$ iff $x \in M \in \mu \Rightarrow M \cap A \neq \emptyset$ and that $c_{\mu}(X \backslash A)=X \backslash i_{\mu}(A)$.

\section{2. $(\mu, \lambda)_{\theta}$-irresolute and related functions}

Definition 1. [18] Let $A$ be a subset of a $\operatorname{GTS}(X, \mu)$. Then

(i) the $\mu_{\theta}$-closure of the set $A$ denoted by $c_{\mu(\theta)}(A)$ is defined as $c_{\mu(\theta)}(A)=$ $\left\{x \in X: c_{\mu}(V) \cap A \neq \varnothing\right.$ for every $\mu$-open set $V$ containing $\left.x\right\}$,

(ii) the $\mu_{\theta}$-interior of $A$ denoted by $i_{\mu(\theta)}(A)$ is defined as $i_{\mu(\theta)}(A)=\{x \in X$ : there exists a $\mu$-open set $G$ containing $x$ such that $x \in G \subseteq c_{\mu}(G) \subseteq A$,

(iii) if $A=c_{\mu(\theta)}(A)$, then $A$ is called $\mu(\theta)$-closed. The complement of a $\mu(\theta)$-closed set is called a $\mu(\theta)$-open set.

Definition 2. A function $f:(X, \mu) \rightarrow(Y, \lambda)$ is said to be $(\mu, \lambda)_{\theta^{-}}$ irresolute at $x \in X$ if for each $\lambda$-open set $V$ containing $f(x)$, there exists a $\mu$-open set $U$ containing $x$ such that $f\left(c_{\mu}(U)\right) \subseteq c_{\lambda}(V)$. The function $f:(X, \mu) \rightarrow(Y, \lambda)$ is said to be $(\mu, \lambda)_{\theta}$-irresolute if it is $(\mu, \lambda)_{\theta}$-irresolute at each point of $X$.

Theorem 1. A function $f:(X, \mu) \rightarrow(Y, \lambda)$ is $(\mu, \lambda)_{\theta}$-irresolute at $x \in X$ if and only if for each $\lambda$-open set $V$ containing $f(x), x \in i_{\mu(\theta)}\left(f^{-1}\left(c_{\lambda}(V)\right)\right)$.

Proof. First let $f$ be $(\mu, \lambda)_{\theta}$-irresolute at $x \in X$ and $V$ be a $\lambda$-open set containing $f(x)$. Then there exists a $\mu$-open set $U$ containing $x$ such that $f\left(c_{\mu}(U)\right) \subseteq c_{\lambda}(V)$. Thus, we have $x \in U \subseteq c_{\mu}(U) \subseteq f^{-1}\left(c_{\lambda}(V)\right)$, and hence, $x \in i_{\mu(\theta)}\left(f^{-1}\left(c_{\lambda}(V)\right)\right)$.

Conversely, let $V \in \lambda$ be such that $x \in i_{\mu(\theta)}\left(f^{-1}\left(c_{\lambda}(V)\right)\right)$ and $f(x) \in V$. Then there exists a $\mu$-open set $U$ such that $x \in U \subseteq c_{\mu}(U) \subseteq f^{-1}\left(c_{\lambda}(V)\right)$, and hence $f\left(c_{\mu}(U)\right) \subseteq c_{\lambda}(V)$. Thus $f$ is $(\mu, \lambda)_{\theta}$-irresolute at $x$.

Theorem 2. A function $f:(X, \mu) \rightarrow(Y, \lambda)$ is $(\mu, \lambda)_{\theta}$-irresolute if and only if for every $\lambda$-open set $V$ of $Y, f^{-1}(V) \subseteq i_{\mu(\theta)}\left(f^{-1}\left(c_{\lambda}(V)\right)\right)$. 
Proof. First let $V$ be any $\lambda$-open set of $Y$ and $x \in f^{-1}(V)$. Then $f(x) \in$ $V$. Now by $(\mu, \lambda)_{\theta}$-irresoluteness of $f, x \in i_{\mu(\theta)}\left(f^{-1}\left(c_{\lambda}(V)\right)\right)$, and hence $f^{-1}(V) \subseteq i_{\mu(\theta)}\left(f^{-1}\left(c_{\lambda}(V)\right)\right)$.

Conversely, let $x \in X$ and $V$ be any $\lambda$-open set of $Y$ containing $f(x)$. Then by hypothesis, $x \in f^{-1}(V) \subseteq i_{\mu(\theta)}\left(f^{-1}\left(c_{\lambda}(V)\right)\right)$. Thus by Theorem 1 , $f$ is $(\mu, \lambda)_{\theta}$-irresolute at $x$.

Theorem 3. For a function $f:(X, \mu) \rightarrow(Y, \lambda)$, the following are equivalent:

(i) $f$ is $(\mu, \lambda)_{\theta}$-irresolute;

(ii) for every subset $A$ of $X, f\left(c_{\mu(\theta)}(A)\right) \subseteq c_{\lambda(\theta)}(f(A))$;

(iii) for every subset $B$ of $Y, c_{\mu(\theta)}\left(f^{-1}(B)\right) \subseteq f^{-1}\left(c_{\lambda(\theta)}(B)\right)$.

Proof. $(i) \Rightarrow(i i)$ : Let $A$ be any subset of $X$. Suppose that $x \in c_{\mu(\theta)}(A)$ and $V$ be any $\lambda$-open set containing $f(x)$. Then there exists a $\mu$-open set $U$ containing $x$ such that $f\left(c_{\mu}(U)\right) \subseteq c_{\lambda}(V)$ (as $f$ is $(\mu, \lambda)_{\theta}$-irresolute). Then obviously, $c_{\mu}(U) \cap A \neq \varnothing$. Thus $\varnothing \neq f\left(c_{\mu}(U)\right) \cap f(A) \subseteq c_{\lambda}(V) \cap f(A)$. Hence $c_{\lambda}(V) \cap f(A) \neq \varnothing$, which implies that $f(x) \in c_{\lambda(\theta)}(f(\bar{A}))$.

$($ ii $) \Rightarrow($ iii $)$ : Let $B$ be any subset of $Y$. Then by hypothesis, $f\left(c_{\mu(\theta)}\left(f^{-1}(B)\right)\right)$ $\subseteq c_{\lambda(\theta)}\left(f\left(f^{-1}(B)\right)\right) \subseteq c_{\lambda(\theta)}(B)$. Thus $c_{\mu(\theta)}\left(f^{-1}(B)\right) \subseteq f^{-1}\left(c_{\lambda(\theta)}(B)\right)$.

(iii) $\Rightarrow(i)$ : Let $x \in X$ and $V$ be any $\lambda$-open set containing $f(x)$. We first note that $f(x) \notin c_{\lambda(\theta)}\left(Y \backslash c_{\lambda}(V)\right)$ (as $\left.c_{\lambda}(V) \cap\left(Y \backslash c_{\lambda}(V)\right)=\varnothing\right)$. Hence $x \notin f^{-1}\left(c_{\lambda(\theta)}\left(Y \backslash c_{\lambda}(V)\right)\right)$. Thus by hypothesis, $x \notin c_{\mu(\theta)}\left(f^{-1}\left(Y \backslash c_{\lambda}(V)\right)\right)$. Consequently, there exists a $\mu$-open set $U$ containing $x$ such that $c_{\mu}(U) \cap$ $\left(f^{-1}\left(Y \backslash c_{\lambda}(V)\right)\right)=\varnothing$. Hence $f\left(c_{\mu}(U)\right) \cap\left(Y \backslash c_{\lambda}(V)\right)=\varnothing$. Thus $f\left(c_{\mu}(U)\right) \subseteq$ $c_{\lambda}(V)$ showing $f$ to be $(\mu, \lambda)_{\theta}$-irresolute.

Theorem 4. A function $f:(X, \mu) \rightarrow(Y, \lambda)$ is $(\mu, \lambda)_{\theta}$-irresolute if and only if for every $\lambda$-open set $V$ of $Y, c_{\mu(\theta)}\left(f^{-1}(V)\right) \subseteq f^{-1}\left(c_{\lambda(\theta)}(V)\right)$.

Proof. One part of the theorem is obvious from $(i) \Rightarrow($ iii $)$ of Theorem 3. The other part is similar to the proof of Theorem $3(i i i) \Rightarrow(i)$.

Definition 3. A GTS $(X, \mu)$ is said to be $\mu$-regular [17] if for each $\mu$ closed set $F$ of $X$ not containing $x$, there exist disjoint $\mu$-open sets $U$ and $V$ such that $x \in U$ and $F \subseteq V$.

Theorem 5. [17] A GTS $(X, \mu)$ is $\mu$-regular if and only if for each $x \in X$ and each $U \in \mu$ containing $x$ there exists $V \in \mu$ containing $x$ such that $x \in V \subseteq c_{\mu}(V) \subseteq U$.

Definition 4. A function $f:(X, \mu) \rightarrow(Y, \lambda)$ is said to be

(a) $(\mu, \lambda)$-continuous [1] if for each $x \in X$ and each $V \in \lambda$ containing $f(x)$, there exists $U \in \mu$ containing $x$ such that $f(U) \subseteq V$;

(b) weakly $(\mu, \lambda)$-continuous [10] if for each $x \in X$ and each $V \in \lambda$ containing $f(x)$, there exists $U \in \mu$ containing $x$ such that $f(U) \subseteq c_{\lambda}(V)$. 
Remark 1. It follows from Definitions 2 and 4 that $(\mu, \lambda)$ continuous $\Rightarrow$ $(\mu, \lambda)_{\theta}$-irresolute $\Rightarrow$ weakly $(\mu, \lambda)$-continuous. But none of the implications is reversible as shown by the examples below.

Example 1. (a) Let $X=\{a, b, c\}, \mu=\lambda=\{\varnothing,\{a\},\{a, b\},\{a, c\}, X\}$. Consider the function $f:(X, \mu) \rightarrow(X, \lambda)$ defined by $f(a)=b, f(b)=c$ and $f(c)=a$. It can be checked easily that $f$ is a $(\mu, \lambda)_{\theta}$-irresolute function but not a $(\mu, \lambda)$-continuous function.

(b) Let $X$ be the set of real numbers, $\mu=\{A: 1 \in A\} \cup\{\varnothing\}, Y=\{a, b, c\}$ and $\lambda=\{\varnothing,\{a\},\{b\},\{a, b\}, Y\}$. Consider the mapping $f:(X, \mu) \rightarrow(Y, \sigma)$ defined by $f(x)=a$ if $x>1$, by $f(x)=b$ if $x<1$ and $f(x)=c$ if $x=1$. It can be verified easily that $f(x)$ is weakly $(\mu, \lambda)$-continuous but not $(\mu, \lambda)_{\theta^{-}}$ irresolute.

Theorem 6. Let $f:(X, \mu) \rightarrow(Y, \lambda)$ be a function such that $(Y, \lambda)$ is $\lambda$-regular. Then the following are equivalent:

(i) $f$ is $(\mu, \lambda)$-continuous;

(ii) $f$ is $(\mu, \lambda)_{\theta}$-irresolute;

(iii) $f$ is weakly $(\mu, \lambda)$-continuous.

Proof. $(i) \Rightarrow(i i)$ : Let $V$ be any $\lambda$-open set containing $f(x)$. Then $f^{-1}\left(c_{\lambda}(V)\right)=c_{\mu}\left(f^{-1}\left(c_{\lambda}(V)\right)\right)$ (as $f^{-1}\left(c_{\lambda}(V)\right)$ is a $\mu$-closed set by (i)). Hence $f^{-1}(V)$ is $\mu$-open. Put $U=f^{-1}(V)$, then $x \in U \subseteq f^{-1}\left(c_{\lambda}(V)\right)$

$=c_{\mu}\left(f^{-1}\left(c_{\lambda}(V)\right)\right)$. This shows that $c_{\mu}(U) \subseteq c_{\mu}\left(f^{-1}\left(c_{\lambda}(V)\right)\right)=f^{-1}\left(c_{\lambda}(V)\right)$. Thus, $f$ is $(\mu, \lambda)_{\theta}$-irresolute.

(ii) $\Rightarrow($ iii $)$ : This is obvious.

$($ iii $) \Rightarrow(i)$ : Let $x \in X$ and $V$ be any $\lambda$-open set in $Y$ containing $f(x)$. Since $(Y, \lambda)$ is $\lambda$-regular, there exists a $\lambda$-open set $W$ such that $f(x) \in W \subseteq$ $c_{\lambda}(W) \subseteq V$. Since $f$ is weakly $(\mu, \lambda)$-continuous, there exists a $\mu$-open set $\bar{U}$ containing $x$ such that $f(U) \subseteq c_{\lambda}(W) \subseteq V$. Hence $f$ is $(\mu, \lambda)$-continuous.

Theorem 7. For a $\mu$-regular space $(X, \mu), f:(X, \mu) \rightarrow(Y, \lambda)$ is $(\mu, \lambda)_{\theta}{ }^{-}$ irresolute if and only if $f$ is weakly $(\mu, \lambda)$-continuous.

Proof. Suppose that $f$ is weakly $(\mu, \lambda)$-continuous. Let $x \in X$ and $V$ be a $\lambda$-open set containing $f(x)$. Then there exists a $\mu$-open set $G$ containing $x$ such that $f(G) \subseteq c_{\lambda}(V)$. Since $(X, \mu)$ is $\mu$-regular, there exists a $\mu$-open set $U$ such that $x \in U \subseteq c_{\mu}(U) \subseteq G$. Therefore, we have $f\left(c_{\mu}(U)\right) \subseteq c_{\lambda}(V)$. Thus, $f$ is $(\mu, \lambda)_{\theta}$-irresolute.

The converse is obvious.

\section{Properties of $(\mu, \lambda)_{\theta}$-irresolute functions}

Definition 5. A GTS $(X, \mu)$ is said to be $\mu$-Urysohn [18] if for any pair of distinct points $x, y \in X$, there exist two $\mu$-open sets $U$ and $V$ such that $x \in U, y \in V$ and $c_{\mu}(U) \cap c_{\mu}(V)=\varnothing$. 
Theorem 8. If for any two distinct points $x_{1}, x_{2} \in X$ and for $a \lambda$ Urysohn space $(Y, \lambda)$, there exists a function $f:(X, \mu) \rightarrow(Y, \lambda)$ such that

(i) $f\left(x_{1}\right) \neq f\left(x_{2}\right)$,

(ii) $f$ is $(\mu, \lambda)_{\theta}$-irresolute at $x_{1}$ and $x_{2}$,

then $(X, \mu)$ is also $\mu$-Urysohn.

Proof. Let $x_{1}$ and $x_{2}$ be two distinct points of $X$. By hypothesis there exists a function $f:(X, \mu) \rightarrow(Y, \lambda)$ satisfying the above two conditions. Let $y_{i}=f\left(x_{i}\right)$, for $i=1,2$. Then $y_{1} \neq y_{2}$. Then there exist $\lambda$-open sets $V_{1}$ and $V_{2}$ containing $y_{1}$ and $y_{2}$ respectively such that $c_{\lambda}\left(V_{1}\right) \cap c_{\lambda}\left(V_{2}\right)=\varnothing$. Again, there exist $\mu$-open sets $U_{1}$ and $U_{2}$ containing $x_{1}$ and $x_{2}$ respectively such that $f\left(c_{\mu}\left(U_{1}\right)\right) \subseteq c_{\lambda}\left(V_{1}\right)$ and $f\left(c_{\mu}\left(U_{2}\right)\right) \subseteq c_{\lambda}\left(V_{2}\right)$. Then $c_{\mu}\left(U_{1}\right) \cap c_{\mu}\left(U_{2}\right)=\varnothing$. Thus, $(X, \mu)$ is $\mu$-Urysohn.

Corollary 1. If $f:(X, \mu) \rightarrow(Y, \lambda)$ is a $(\mu, \lambda)_{\theta}$-irresolute injection and $(Y, \lambda)$ is $\lambda$-Urysohn, then $(X, \mu)$ is also $\mu$-Urysohn.

Definition 6. A function $f:(X, \mu) \rightarrow(Y, \lambda)$ is said to have strongly $\mu_{\theta}$-closed graph if for each $(x, y) \in X \times Y \backslash G(f)$, there exist a $\mu$-open set $U$ containing $x$ and a $\lambda$-open set $V$ of $Y$ containing $y$ such that $\left[c_{\mu}(U) \times\right.$ $\left.c_{\lambda}(V)\right] \cap G(f)=\varnothing$.

Lemma 1. A function $f:(X, \mu) \rightarrow(Y, \lambda)$ has strongly $\mu_{\theta}$-closed graph if and only if for each $(x, y) \in X \times Y \backslash G(f)$, there exist a $\mu$-open set $U$ in $X$ containing $x$ and $a \lambda$-open set $V$ of $Y$ containing $y$ such that $f\left(c_{\mu}(U)\right) \cap$ $c_{\lambda}(V)=\varnothing$.

Proof. Easy.

Theorem 9. If $f:(X, \mu) \rightarrow(Y, \lambda)$ is a $(\mu, \lambda)_{\theta}$-irresolute function and $(Y, \lambda)$ is $\lambda$-Urysohn, then $G(f)$ is strongly $\mu_{\theta}$-closed.

Proof. Suppose that $(x, y) \in X \times Y \backslash G(f)$. Then $y \neq f(x)$. Since $Y$ is $\lambda$-Urysohn, there exist $\lambda$-open sets $V$ and $W$ in $Y$ containing $y$ and $f(x)$ respectively such that $c_{\lambda}(V) \cap c_{\lambda}(W)=\varnothing$. Since $f$ is $(\mu, \lambda)_{\theta}$-irresolute, there exists a $\mu$-open set containing $x$ such that $f\left(c_{\mu}(U)\right) \subseteq c_{\lambda}(W)$. Thus, $f\left(c_{\mu}(U)\right) \cap c_{\lambda}(V)=\varnothing$, and hence by Lemma $1, G(f)$ is strongly $\mu_{\theta}$-closed.

Theorem 10. If $f:(X, \mu) \rightarrow(Y, \lambda)$ is an injective $(\mu, \lambda)_{\theta}$-irresolute function with a strongly $\mu_{\theta}$-closed graph, then $(X, \mu)$ is $\mu$-Urysohn.

Proof. Let $x$ and $y$ be two distinct points of $X$. Then, since $f$ is injective, $f(x) \neq f(y)$. Hence $(x, f(y)) \in X \times Y \backslash G(f)$. Since $G(f)$ is strongly $\mu_{\theta^{-}}$ closed, by Lemma 1, there exist a $\mu$-open set $U$ of $X$ containing $x$ and a $\lambda$-open set $V$ of $Y$ containing $f(y)$ such that $f\left(c_{\mu}(U)\right) \cap c_{\lambda}(V)=\varnothing$. Since $f$ is $(\mu, \lambda)_{\theta}$-irresolute, there exists a $\mu$-open set $G$ containing $y$ such that 
$f\left(c_{\mu}(G)\right) \subseteq c_{\lambda}(V)$. Therefore, $f\left(c_{\mu}(U)\right) \cap f\left(c_{\mu}(G)\right)=\varnothing$. Thus, $c_{\mu}(U) \cap$ $c_{\mu}(G)=\varnothing$. Hence $(X, \mu)$ is $\mu$-Urysohn.

Definition 7. A subset $A$ of a $\operatorname{GTS}(X, \mu)$ is said to be weakly $\mu$-compact relative to $X$ [19] if for any cover $\left\{U_{\alpha}: \alpha \in \Lambda\right\}$ of $A$ by $\mu$-open sets of $X$, there exists a finite subset $\Lambda_{0}$ of $\Lambda$ such that $A \subseteq \cup\left\{c_{\mu}\left(U_{\alpha}\right): \alpha \in \Lambda_{0}\right\}$. If $A=X$, then $(X, \mu)$ is said to be weakly $\mu$-compact.

Theorem 11. If $f:(X, \mu) \rightarrow(Y, \lambda)$ is a $(\mu, \lambda)_{\theta}$-irresolute function and $K$ is weakly $\mu$-compact relative to $(X, \mu)$, then $f(K)$ is weakly $\lambda$-compact relative to $(Y, \lambda)$.

Proof. Let $K$ be a $\mu$-closed set relative to $X$. Let $\left\{V_{\alpha}: \alpha \in \Lambda\right\}$ be any cover of $f(K)$ by $\lambda$-open sets of $(Y, \lambda)$. For each $x \in K$, there exists $\alpha(x) \in \Lambda$ such that $f(x) \in V_{\alpha(x)}$. Since $f$ is $(\mu, \lambda)_{\theta}$-irresolute, there exists a $\mu$-open set $U(x)$ containing $x$ such that $f\left(c_{\mu}(U(x))\right) \subseteq c_{\lambda}\left(V_{\alpha(x)}\right)$. Then the family $\{U(x): x \in K\}$ is a cover of $K$ by $\mu$-open sets of $X$. Since $K$ is weakly $\mu$-compact relative to $(X, \mu)$, there exists a finite number of points, say, $x_{1}, x_{2}, \ldots, x_{n}$ in $K$ such that $K \subseteq \cup\left\{c_{\mu}\left(U\left(x_{k}\right)\right): x_{k} \in K, 1 \leq k \leq n\right\}$. Therefore, we obtain $f(K) \subseteq \cup\left\{f\left(c_{\mu}\left(U\left(x_{k}\right)\right)\right): x_{k} \in K, 1 \leq k \leq n\right\} \subseteq$ $\cup\left\{c_{\lambda}\left(V_{\alpha(x)}\right): x_{k} \in K, 1 \leq k \leq n\right\}$. Thus, $f(K)$ is $\lambda$-closed relative to $(Y, \lambda)$.

Corollary 2. If $f:(X, \mu) \rightarrow(Y, \lambda)$ is a $(\mu, \lambda)_{\theta}$-irresolute surjection and $(X, \mu)$ is weakly $\mu$-compact, then $(Y, \lambda)$ is weakly $\lambda$-compact.

Definition 8. Let $(X, \mu)$ be a GTS. The $\mu(\theta)$-frontier of $A$ denoted by $\operatorname{Fr}_{\mu(\theta)}(A)$ is defined as $\operatorname{Fr}_{\mu(\theta)}(A)=c_{\mu(\theta)}(A) \cap c_{\mu(\theta)}(X \backslash A)$.

Theorem 12. The set of all points $x \in X$ at which a function $f$ : $(X, \mu) \rightarrow(Y, \lambda)$ is not $(\mu, \lambda)_{\theta}$-irresolute is identical with the union of the $\mu(\theta)$-frontiers of the inverse images of the $\lambda$-closures of $\lambda$-open sets containing $f(x)$.

Proof. Suppose that $f$ is not $(\mu, \lambda)_{\theta}$-irresolute at $x \in X$. Then there exists a $\lambda$-open set $V$ of $Y$ containing $f(x)$ such that $f\left(c_{\mu}(U)\right)$ is not contained in $c_{\lambda}(V)$, for every $\mu$-open set $U$ containing $x$. Then $c_{\mu}(U) \cap\left(X \backslash f^{-1}\left(c_{\lambda}(V)\right)\right) \neq$ $\varnothing$, for every $\mu$-open set $U$ containing $x$, and hence $x \in c_{\mu(\theta)}\left(X \backslash f^{-1}\left(c_{\lambda}(V)\right)\right)$. On the other hand, we have $x \in f^{-1}(V) \subseteq c_{\mu(\theta)}\left(f^{-1}\left(c_{\lambda}(V)\right)\right)$, and hence $x \in \operatorname{Fr}_{\mu(\theta)}\left(f^{-1}\left(c_{\lambda}(V)\right)\right)$.

Conversely, suppose that $f$ is $(\mu, \lambda)_{\theta}$-irresolute at $x \in X$ and let $V$ be any $\lambda$-open set containing $f(x)$. Then by Theorem 2, we have $x \in f^{-1}(V) \subseteq$ $i_{\mu(\theta)}\left(f^{-1}\left(c_{\lambda}(V)\right)\right)$. Thus, $x \notin F r_{\mu(\theta)}\left(f^{-1}\left(c_{\lambda}(V)\right)\right)$ for every $\lambda$-open set $V$ containing $f(x)$. 


\section{Acknowledgements}

The author acknowledges the financial support from Department of Higher education, Science and technology and Biotechnology, Government of West Bengal, INDIA.

\section{References}

[1] Á. Császár, Generalized topology, generalized continuity, Acta Math. Hungar. 96 (2002), 351-357.

[2] Á. Császár, Generalized open sets in generalized topologies, Acta Math. Hungar. 106 (2005), 53-66.

[3] Á. Császár, Normal generalized topologies, Acta Math. Hungar. 115 (2007), 309-313.

[4] Á. Császár, $\delta$ - and $\theta$-modifications of generalized topologies, Acta Math. Hungar. 120 (2008), 275-279.

[5] G. Di Maio and T. Noiri, Weak and strong forms of irresolute functions, Suppl. Rend. Circ. Mat. Palermo 18(2) (1988), 255-273.

[6] K. K. Dube, G. I. Chae, and O. S. Panwar, On mappings with strongly semi-closed graphs, Ulsan Inst. Tech. Rep. 15 (1984), 373-379.

[7] M. S. Espelie and J. E. Joseph, Remarks on two weak forms of continuity, Canad. Math. Bull. 25 (1982), 59-63.

[8] S. Fomin, Extensions of topological spaces, Ann. Math., 44 (1943), 471-480.

[9] F. H. Khedr and T. Noiri, On $\theta$-irresolute functions, Indian J. Math. 28(3) (1986) $211-217$.

[10] W. K. Min, Weak continuity in generalized topological spaces, Acta Math. Hungar. 124 (2009), 73-81.

[11] T. Noiri, Between continuity and weak-continuity, Boll. Un. Mat. Ital. 9(4) (1974), 647-654.

[12] T. Noiri, Properties of $\theta$-continuous functions, Atti Accad. Naz. Lincei. Rend. Cl. Sci. Fiz. Mat. Nat. 58(8) (1975), 887-891.

[13] T. Noiri, On 6-preirresolute functions, Acta Math. Hungar. 95 (2002), 287-297.

[14] T. Noiri, Weak and strong forms of $\beta$-irresolute functions, Acta Math. Hungar. 99 (2003), 305-318.

[15] T. Noiri, Unified characterazitions for modification of $R_{0}$ and $R_{1}$ topological spaces, Rend. Circ. Mat. Palermo. 55(2) (2006), 29-42.

[16] V. Popa, Characterizations of $\theta$-continuous functions, Stud. Cerc. St. Ser. Mat. 32 (1980), 113-119.

[17] B. Roy, On a type of generalized open sets, Appl. Gen. Topol. 12(2) (2011), 163-173.

[18] B. Roy and T. Noiri, Unification on strongly $\theta$-continuous functions, An. Univ. Vest Timis. Ser. Mat.-Inform. 51(2) (2013), 95-103.

[19] M. S. Sarsak, Weakly $\mu$-compact spaces, Demonstratio Math. 45(4) (2012), 929-938.

Department of Mathematics, Women's Christian College, 6, Greek Church Row, KolKatA-700 026, INDIA

E-mail address: bishwambhar_roy@yahoo.co.in 